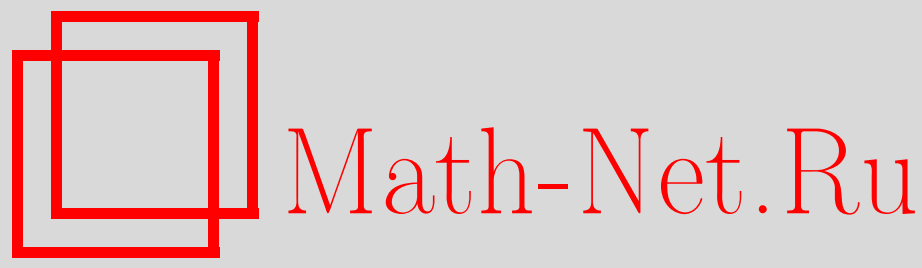

Т. Х. Расулов, Исследование спектра одного модельного оператора в пространстве Фока, ТМФ, 2009, том 161, номер 2, 164-175

DOI: https://doi.org/10.4213/tmf6429

Использование Общероссийского математического портала Math-Net.Ru подразумевает, что вы прочитали и согласны с пользовательским соглашением http://www.mathnet.ru/rus/agreement

Параметры загрузки:

IP: 52.23 .180 .231

26 апреля 2023 г., 16:26:54

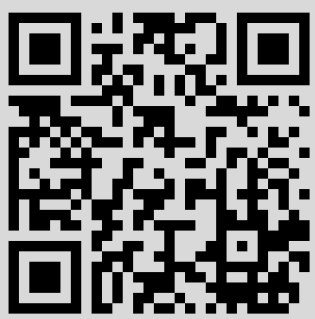




\title{
ИССЛЕДОВАНИЕ СПЕКТРА ОДНОГО МОДЕЛЬНОГО OПЕРАТОРА В ПРОСТРАНСТВЕ ФОКА
}

\begin{abstract}
Рассмотрен модельный оператор $H$, соответствующий квантовой системе с несохраняющимся ограниченным числом частиц на решетке. На основе анализа спектра канальных операторов описано положение существенного спектра оператора $H$. Получено уравнение типа уравнения Фаддеева для собственных векторов оператора $H$.
\end{abstract}

Ключевые слова: модельный оператор, система с несохраняющимся ограниченным числом частиц, уравнение Фаддеева, существенный спектр, канальный оператор.

\section{1. ВВЕДЕНИЕ}

Исследованию существенного спектра системы с сохраняющимся ограниченным числом частиц в непрерывном пространстве и на решетке посвящены многие работы (см., например, [1], [2] и [3], [4] соответственно). Отметим, что в непрерывном пространстве и на решетке имеются более интересные в определенном смысле задачи, в которых число частиц не сохраняется. Такие задачи возникают в физике твердого тела, квантовой теории поля и статистической физике. Системы с несохраняющимся ограниченным числом частиц в непрерывном пространстве рассмотрены в работах [5], [6].

В настоящей работе рассматривается (решетчатый) модельный оператор $H$, действующий в трехчастичном обрезанном подпространстве пространства Фока. Выделены канальные операторы, и положение существенного спектра оператора $H$ описано через спектры канальных операторов. Также получен аналог уравнения Фаддеева для собственных векторов оператора $H$.

Заметим, что ранее в работе [7] оператор $H$ рассмотрен в случае, когда операторы $K_{i}, i=1,2$, определенные ниже, являются частичными интегральными операторами с вырожденными ядрами, а в работе [8] - в случае, когда $K_{i}=0, i=1,2$. Существенный и дискретный спектры, а также уравнение Фаддеева для симметричного варианта модельного оператора $H$ изучены в работах [9]-[14] (в случаях, когда $K_{i}, i=1,2$, являются частичными интегральными операторами с вырожденными

* Самаркандский государственный университет, Самарканд, Узбекистан. E-mail: rth@mail.ru 
и невырожденными ядрами, а также нулевыми операторами). Оператор $H$, pacсматриваемый в настоящей работе, и симметричный вариант оператора $H$ имеют разные спектральные свойства, поэтому исследование спектральных свойств нашего модельного оператора играет важную роль в современной математической физике.

Структура работы следующая. В разделе 2 модельный оператор рассматривается как ограниченный самосопряженный оператор в гильбертовом пространстве и формулируется основной результат работы. В разделе 3 , пользуясь разложением в прямой операторный интеграл, мы сводим изучение спектральных свойств модельного оператора к изучению спектральных свойств семейства операторов обобщенной модели Фридрихса. В разделе 4 получен аналог уравнения Фаддеева для собственных векторов оператора $H$. Раздел 5 посвящен доказательству основного результата настоящей работы.

\section{2. МОДЕЛЬНЫЙ ОПЕРАТОР И ФОРМУЛИРОВКА ОСНОВНОГО РЕЗУЛЬТАТА}

Пусть $\mathbf{T}^{\nu} \equiv(-\pi ; \pi]^{\nu}-\nu$-мерный куб с соответствующим отождествлением противоположных граней, $\left(\mathbf{T}^{\nu}\right)^{2}=\mathbf{T}^{\nu} \times \mathbf{T}^{\nu}-$ декартово произведение, $L_{2}\left(\left(\mathbf{T}^{\nu}\right)^{n}\right)-$ гильбертово пространство квадратично-интегрируемых (комплекснозначных) функций, определенных на $\left(\mathbf{T}^{\nu}\right)^{n}, n=1,2, \mathbb{C}$ - комплексная плоскость. Положим

$$
\mathcal{H}_{0}=\mathbb{C}, \quad \mathcal{H}_{n}=L_{2}\left(\left(\mathbf{T}^{\nu}\right)^{n}\right), \quad n=1,2, \quad \mathcal{H}^{(n, m)}=\bigoplus_{i=n}^{m} \mathcal{H}_{i}, \quad 0 \leqslant n<m \leqslant 2 .
$$

Рассмотрим модельный оператор $H$, действующий в гильбертовом пространстве $\mathcal{H}^{(0,2)}$ и заданный как операторная матрица

$$
H=\left(\begin{array}{ccc}
H_{00} & H_{01} & 0 \\
H_{10} & H_{11} & H_{12} \\
0 & H_{21} & H_{22}
\end{array}\right)
$$

где операторы $H_{i j}: \mathcal{H}_{j} \rightarrow \mathcal{H}_{i}, i, j=0,1,2$, определяются формулами

$$
\begin{gathered}
\left(H_{00} f_{0}\right)_{0}=w_{0} f_{0}, \quad\left(H_{01} f_{1}\right)_{0}=\int v_{1}(s) f_{1}(s) d s, \quad\left(H_{10} f_{0}\right)_{1}(p)=v_{1}(p) f_{0} \\
\left(H_{11} f_{1}\right)_{1}(p)=w_{1}(p) f_{1}(p), \quad\left(H_{12} f_{2}\right)_{1}(p)=\int v_{2}(s) f_{2}(p, s) d s \\
\left(H_{21} f_{1}\right)_{2}(p, q)=v_{2}(q) f_{1}(p), \\
H_{22}=H_{22}^{0}-K_{1}-K_{2}, \quad\left(H_{22}^{0} f_{2}\right)_{2}(p, q)=w_{2}(p, q) f_{2}(p, q), \\
\left(K_{1} f_{2}\right)_{2}(p, q)=\int K_{1}(p, s) f_{2}(s, q) d s, \quad\left(K_{2} f_{2}\right)_{2}(p, q)=\int K_{2}(s, q) f_{2}(p, s) d s
\end{gathered}
$$

Здесь $f_{i} \in \mathcal{H}_{i}, i=0,1,2 ; w_{0}$ - фиксированное вещественное число; $w_{1}(\cdot), v_{i}(\cdot)-$ вещественнозначные непрерывные функции на $\mathbf{T}^{\nu}, w_{2}(\cdot, \cdot)$ и $K_{i}(\cdot, \cdot)$ - вещественнозначные непрерывные функции на $\left(\mathbf{T}^{\nu}\right)^{2}, i=1,2$. Здесь и далее интеграл без 
указания пределов означает интегрирование по всей области изменения переменных интегрирования.

В дальнейшем мы будем дополнительно предполагать, что операторы $K_{1}$ и $K_{2}$ являются положительными и принадлежат пространству операторов со следом. При таких предположениях существуют положительные корни из этих операторов, задающиеся формулами

$$
\begin{aligned}
& \left(K_{1}^{1 / 2} f_{2}\right)(p, q)=\int K_{1}^{1 / 2}(p, s) f_{2}(s, q) d s \\
& \left(K_{2}^{1 / 2} f_{2}\right)(p, q)=\int K_{2}^{1 / 2}(s, q) f_{2}(p, s) d s
\end{aligned}
$$

где через $K_{i}^{1 / 2}(\cdot, \cdot)$ формально обозначено ядро оператора $K_{i}^{1 / 2}, i=1,2$. В математической физике операторы $H_{01}$ и $H_{12}$ называются операторами уничтожения, а операторы $H_{10}$ и $H_{21}$ - операторами рождения.

Можно легко проверить, что оператор $H$, определенный операторной матрицей $(2.1)$ и действующий в гильбертовом пространстве $\mathcal{H}^{(0,2)}$, является ограниченным и самосопряженным.

Для формулировки основного результата работы наряду с оператором $H$ рассмотрим также операторы $H_{1}$ и $H_{2}$, действующие соответственно в гильбертовых пространствах $\mathcal{H}_{2}$ и $\mathcal{H}^{(1,2)}$ по формулам

$$
H_{1}=H_{22}^{0}-K_{1}, \quad H_{2}=\left(\begin{array}{cc}
H_{11} & H_{12} \\
H_{21} & H_{22}^{0}-K_{2}
\end{array}\right),
$$

и семейство операторов обобщенной модели Фридрихса $h_{1}(q), q \in \mathbf{T}^{\nu}$, и $h_{2}(p)$, $p \in \mathbf{T}^{\nu}$, действующих соответственно в $\mathcal{H}_{1}$ и $\mathcal{H}^{(0,1)}$ по формулам

$$
h_{1}(q)=h_{1}^{(0)}(q)-k_{1}, \quad h_{2}(p)=\left(\begin{array}{cc}
h_{00}^{(2)}(p) & h_{01}^{(2)} \\
h_{10}^{(2)} & h_{11}^{(2,0)}(p)-k_{2}
\end{array}\right),
$$

где элементы задаются следующим образом:

$$
\begin{gathered}
\left(h_{1}^{(0)}(q) f_{1}\right)(p)=w_{2}(p, q) f_{1}(p), \quad\left(k_{1} f_{1}\right)(p)=\int K_{1}(p, s) f_{1}(s) d s, \quad f_{1} \in \mathcal{H}, \\
\left(h_{00}^{(2)}(p) f_{0}\right)_{0}=w_{1}(p) f_{0}, \quad\left(h_{01}^{(2)} f_{1}\right)_{0}=\int v_{2}(s) f_{1}(s) d s, \\
\left(h_{10}^{(2)} f_{0}\right)_{1}(q)=v_{2}(q) f_{0}, \quad\left(h_{11}^{(2,0)}(p) f_{1}\right)_{1}(q)=w_{2}(p, q) f_{1}(q), \\
\left(k_{2} f_{1}\right)_{1}(q)=\int K_{2}(s, q) f_{1}(s) d s, \quad f_{i} \in \mathcal{H}_{i}, \quad i=0,1 .
\end{gathered}
$$

Очевидно, что операторы $H_{1}$ и $H_{2}$ являются ограниченными и самосопряженными в $\mathcal{H}_{2}$ и $\mathcal{H}^{(1,2)}$ соответственно. Они выбраны единственным образом из некомпактной части оператора $H$ в силу свойства разложимости в прямой операторный интеграл (см. раздел 3). 
Пусть $\sigma\left(H_{i}\right)$ - спектр оператора $H_{i}, i=1,2$, и

$$
m=\min _{p, q \in \mathbf{T}^{\nu}} w_{2}(p, q), \quad M=\max _{p, q \in \mathbf{T}^{\nu}} w_{2}(p, q) .
$$

Введем также обозначения $\sigma_{\text {disc }}(\cdot)$ и $\sigma_{\text {ess }}(\cdot)$ для дискретного и существенного спектров соответственно. Спектр операторов $H_{i}, i=1,2$, описывается следующей теоремой.

Теорема 1. Для спектра $\sigma\left(H_{i}\right)$ оператора $H_{i}$ имеет место равенство

$$
\sigma\left(H_{i}\right)=\sigma_{\text {two }}\left(H_{i}\right) \cup \sigma_{\text {three }}\left(H_{i}\right),
$$

где

$$
\sigma_{\text {two }}\left(H_{i}\right)=\bigcup_{p \in \mathbf{T}^{\nu}} \sigma_{\text {disc }}\left(h_{i}(p)\right), \quad \sigma_{\text {three }}\left(H_{i}\right)=[m, M], \quad i=1,2 .
$$

Теперь сформулируем основной результат настоящей работы - теорему, которая описывает существенный спектр оператора $H$.

Tеорема 2. Существенный спектр $\sigma_{\text {ess }}(H)$ оператора $H$ совпадает с объединением спектров операторов $H_{1}$ u $H_{2}$, m.e. $\sigma_{\mathrm{ess}}(H)=\sigma\left(H_{1}\right) \cup \sigma\left(H_{2}\right)$.

Согласно теореме 2 операторы $H_{1}$ и $H_{2}$ обладают характеристическим свойством канального оператора (см., например, работы [3]-[5], [10]). По этой причине мы назовем их канальными операторами, соответствующими оператору $H$. Заметим, что канальные операторы $H_{1}$ и $H_{2}$ имеют более простую структуру, чем $H$, и поэтому теорема 2 играет важную роль в дальнейших исследованиях спектра оператора $H$.

Теперь введем новые подмножества существенного спектра оператора $H$.

ОПРЕДЕЛЕНИЕ [9], [13], [14]. Множества

$$
\sigma_{\text {two }}(H)=\sigma_{\text {two }}\left(H_{1}\right) \cup \sigma_{\text {two }}\left(H_{2}\right), \quad \sigma_{\text {three }}(H)=\sigma_{\text {three }}\left(H_{1}\right)=\sigma_{\text {three }}\left(H_{2}\right)
$$

называются двухчастичной и трехчастичной ветвями существенного спектра оператора $H$ соответственно.

\section{3. НЕКОТОРЫЕ СПЕКТРАЛЬНЫЕ СВОЙСТВА КАНАЛЬНЫХ ОПЕРАТОРОВ}

В этом разделе, используя разложение в прямой операторный интеграл, мы сводим изучение спектральных свойств канальных операторов $H_{i}$ к изучению спектральных свойств семейства операторов обобщенной модели Фридрихса $h_{i}(p)$, $p \in \mathbf{T}^{\nu}$, которые определены формулами (2.3). Мы описываем спектр операторов $h_{i}(p), p \in \mathbf{T}^{\nu}$, и доказываем теорему 1 .

Сначала рассмотрим оператор $H_{2}$, который коммутирует с любым оператором умножения $U_{\alpha}$ на ограниченную функцию $\alpha(\cdot)$, заданную на $\mathbf{T}^{\nu}$. Оператор $U_{\alpha}$ действует в $\mathcal{H}^{(1,2)}$ по правилу

$$
U_{\alpha}\left(\begin{array}{c}
g_{1}(p) \\
g_{2}(p, q)
\end{array}\right)=\left(\begin{array}{c}
\alpha(p) g_{1}(p) \\
\alpha(p) g_{2}(p, q)
\end{array}\right), \quad\left(\begin{array}{c}
g_{1} \\
g_{2}
\end{array}\right) \in \mathcal{H}^{(1,2)}
$$


Из разложения $\mathcal{H}^{(1,2)}=\int \oplus \mathcal{H}^{(0,1)} d p$ пространства $\mathcal{H}^{(1,2)}$ в прямой интеграл [2] следует, что оператор $H_{2}$ разлагается в прямой интеграл: $H_{2}=\int \oplus h_{2}(p) d p$, где оператор $h_{2}(p)$ определен в $(2.3), p \in \mathbf{T}^{\nu}$.

Пусть оператор $h_{2}^{0}(p), p \in \mathbf{T}^{\nu}$, действует в $\mathcal{H}^{(0,1)}$ как

$$
h_{2}^{0}(p)=\left(\begin{array}{cc}
0 & 0 \\
0 & h_{11}^{(2,0)}(p)
\end{array}\right) \text {. }
$$

Оператор возмущения $h_{2}(p)-h_{2}^{0}(p)$ оператора $h_{2}^{0}(p)$ является самосопряженным компактным оператором. Из известной теоремы Вейля о сохранении существенного спектра при компактных возмущениях вытекает, что существенный спектр оператора $h_{2}(p)$ совпадает с существенным спектром оператора $h_{2}^{0}(p), p \in \mathbf{T}^{\nu}$. Известно, что $\sigma_{\mathrm{ess}}\left(h_{2}^{0}(p)\right)=[m(p), M(p)]$, где числа $m(p)$ и $M(p)$ определяются следующим образом:

$$
m(p)=\min _{q \in \mathbf{T}^{\nu}} w_{2}(p, q), \quad M(p)=\max _{q \in \mathbf{T}^{\nu}} w_{2}(p, q) .
$$

Из последних двух фактов следует, что $\sigma_{\mathrm{ess}}\left(h_{2}(p)\right)=[m(p), M(p)]$.

ЗАмечаниЕ 1. Для некоторого $p \in \mathbf{T}^{\nu}$ существенный спектр оператора $h_{2}(p)$ может стянуться в точку $m(p)$ и, следовательно, мы не можем сказать, что для любого $p \in \mathbf{T}^{\nu}$ существенный спектр оператора $h_{2}(p)$ является абсолютно непрерывным. Например, если функция $w_{2}(\cdot, \cdot)$ имеет вид $w_{2}(p, q)=\varepsilon(p)+\varepsilon(p+q)+\varepsilon(q)$, где

$$
\varepsilon(q)=\sum_{i=1}^{\nu}\left(1-\cos q_{i}\right), \quad q=\left(q_{1}, \ldots, q_{\nu}\right) \in \mathbf{T}^{\nu}, \quad p=(\underbrace{\pi, \ldots, \pi}_{\nu}) \in \mathbf{T}^{\nu},
$$

то $\sigma_{\text {ess }}\left(h_{2}(p)\right)=\{4 \nu\}$.

Докажем два вспомогательных утверждения, которые понадобятся в этом и следующих разделах.

Лемма 1. Операторы $k_{i}, i=1,2$, в формулах (2.3) являются положительными, и положительные квадратные корни $k_{i}^{1 / 2}$ из этих операторов задаются равенствами

$$
\left(k_{1}^{1 / 2} f\right)(p)=\int K_{1}^{1 / 2}(p, s) f(s) d s, \quad\left(k_{2}^{1 / 2} f\right)(q)=\int K_{2}^{1 / 2}(s, q) f(s) d s,
$$

где функиия $K_{i}^{1 / 2}(\cdot, \cdot)$ есть квадратично-интегрируемое ядро оператора $k_{i}^{1 / 2}$.

ДокАЗАтЕЛЬство. Из положительности оператора $K_{i}$ следует, что оператор $k_{i}$ также является положительным. Следовательно, каждое нетривиальное собственное значение $\lambda_{m}^{i}$ оператора $k_{i}$ положительно. В силу теоремы Гильберта-Шмидта имеем разложение $k_{i}=\sum_{m} \lambda_{m}^{i}\left(\varphi_{m}^{i}, \cdot\right) \varphi_{m}^{i}$ с условием $\sum_{m} \lambda_{m}^{i}<\infty$; здесь $\varphi_{m}-$ собственный вектор оператора $k_{i}$, соответствующий собственному значению $\lambda_{m}^{i}$, $i=1,2$. Пусть $k_{i}^{1 / 2}$ есть положительный корень из оператора $k_{i}$, тогда $k_{i}^{1 / 2}=$ $\sum_{m} \sqrt{\lambda_{m}^{i}}\left(\varphi_{m}^{i}, \cdot\right) \varphi_{m}^{i}$. В силу условия $\sum_{m} \lambda_{m}<\infty$ оператор $k_{i}^{1 / 2}$ является оператором Гильберта-Шмидта. Следовательно, ядро $K_{i}^{1 / 2}(\cdot, \cdot)$ интегрального оператора $k_{i}^{1 / 2}$ является квадратично-интегрируемым. Лемма 1 доказана. 
Пусть $I_{i}$ - единичный оператор в $\mathcal{H}_{i}, i=0,1,2$.

Лемма 2. Положительный корень из оператора $K_{i}, i=1,2$, определяется по формуле (2.2).

ДокАЗАтЕльство. Операторы $K_{i}, i=1,2$, представимы в виде $K_{1}=k_{1} \otimes I_{1}$, $K_{2}=I_{1} \otimes k_{2}$. В силу леммы 1 операторы $k_{i}, i=1,2$, являются положительными, и положительные корни из этих операторов определяются по формуле (3.1). Легко можно проверить, что $K_{1}^{1 / 2}=k_{1}^{1 / 2} \otimes I_{1}$ и $K_{2}^{1 / 2}=I_{1} \otimes k_{2}^{1 / 2}$. Лемма 2 доказана.

При каждом фиксированном $p \in \mathbf{T}^{\nu}$ оператор $A_{0}(p ; z), z \in \mathbb{C} \backslash \sigma_{\mathrm{ess}}\left(h_{2}(p)\right)$, действует в $\mathcal{H}^{(0,1)}$ как

$$
A_{0}(p ; z)=\left(\begin{array}{ll}
A_{00}(p ; z) & A_{01}(p ; z) \\
A_{10}(p ; z) & A_{11}(p ; z)
\end{array}\right)
$$

где

$$
\begin{aligned}
\left(A_{00}(p ; z) g_{0}\right)_{0} & =\left(w_{1}(p)-z-\int \frac{v_{2}^{2}(s) d s}{w_{2}(p, s)-z}-1\right) g_{0}, \\
\left(A_{01}(p ; z) g_{1}\right)_{0} & =\int \frac{v_{2}(s) d s}{w_{2}(p, s)-z} \int K_{2}^{1 / 2}(t, s) g_{1}(t) d t \\
\left(A_{10}(p ; z) g_{0}\right)_{1}(q) & =\int \frac{K_{2}^{1 / 2}(s, q) v_{2}(s) d s}{w_{2}(p, s)-z} \cdot g_{0}, \\
\left(A_{11}(p ; z) g_{1}\right)_{1}(q) & =-\int \frac{K_{2}^{1 / 2}(t, q) d t}{w_{2}(p, t)-z} \int K_{2}^{1 / 2}(s, t) g_{1}(s) d s .
\end{aligned}
$$

Отметим, что при любых фиксированных $p \in \mathbf{T}^{\nu}$ и $z \in \mathbb{C} \backslash \sigma_{\mathrm{ess}}\left(h_{2}(p)\right)$ оператор $A_{0}(p ; z)$ принадлежит пространству операторов со следом. Следовательно, $\operatorname{det}\left[I^{(0,1)}+A_{0}(p ; z)\right]$ оператора $I^{(0,1)}+A_{0}(p ; z)$ хорошо определен (здесь $I^{(0,1)}=$ $\left.\operatorname{diag}\left\{I_{0}, I_{1}\right\}\right)$.

Следующая лемма устанавливает связь между собственными значениями оператора $h_{2}(p)$ и нулями функции $\operatorname{det}\left[I^{(0,1)}+A_{0}(p ; \cdot)\right], p \in \mathbf{T}^{\nu}$.

Лемма 3. При каждом фиксированном $p \in \mathbf{T}^{\nu}$ число $z \in \mathbb{C} \backslash \sigma_{\mathrm{ess}}\left(h_{2}(p)\right)$ является собственным значением оператора $h_{2}(p)$ тогда и только тогда, когда $\operatorname{det}\left[I^{(0,1)}+\right.$ $\left.A_{0}(p ; z)\right]=0$.

ДокАзАтельство. Пусть число $z \in \mathbb{C} \backslash \sigma_{\text {ess }}\left(h_{2}(p)\right)$ является собственным значением оператора $h_{2}(p), p \in \mathbf{T}^{\nu}$, и $f=\left(f_{0}, f_{1}\right) \in \mathcal{H}^{(0,1)}$ - соответствующая собственная вектор-функция. Тогда $f_{0}$ и $f_{1}$ удовлетворяют следующей системе уравнений:

$$
\begin{aligned}
\left(w_{1}(p)-z\right) f_{0}+\int v_{2}(s) f_{1}(s) d s & =0, \\
v_{2}(q) f_{0}+\left(w_{2}(p, q)-z\right) f_{1}(q)-\int K_{2}(s, q) f_{1}(s) d s & =0 .
\end{aligned}
$$

Так как $w_{2}(p, q)-z \neq 0$ для любых $z \in \mathbb{C} \backslash \sigma_{\mathrm{ess}}\left(h_{2}(p)\right)$ и $q \in \mathbf{T}^{\nu}$, из второго уравнения системы (3.3) для $f_{1}$ имеем

$$
f_{1}(q)=\frac{\left(k_{2}^{1 / 2} \tilde{f}_{1}\right)(q)}{w_{2}(p, q)-z}-\frac{v_{2}(q) f_{0}}{w_{2}(p, q)-z}
$$


где оператор $k_{2}^{1 / 2}$ определен формулой (3.1) и

$$
\tilde{f}_{1}(q)=\left(k_{2}^{1 / 2} f_{1}\right)(q) .
$$

Подставляя выражение (3.4) для $f_{1}$ в первое уравнение системы (3.3) и равенство (3.5), получим, что система уравнений (3.3) имеет ненулевое решение тогда и только тогда, когда система уравнений

$$
\begin{aligned}
& \left(w_{1}(p)-z-\int \frac{v_{2}^{2}(s) d s}{w_{2}(p, s)-z}\right) f_{0}+\int \frac{v_{2}(s) d s}{w_{2}(p, s)-z} \int K_{2}^{1 / 2}(t, s) \tilde{f}_{1}(t) d t=0 \\
& \int \frac{K_{2}^{1 / 2}(s, q) v_{2}(s) d s}{w_{2}(p, s)-z} \cdot f_{0}+\tilde{f}_{1}(q)-\int \frac{K_{2}^{1 / 2}(t, q) d t}{w_{2}(p, t)-z} \int K_{2}^{1 / 2}(s, t) \tilde{f}_{1}(s) d s=0
\end{aligned}
$$

или, что эквивалентно, уравнение $I^{(0,1)} \Phi+A_{0}(p ; z) \Phi=0$, где $\Phi=\left(f_{0}, \tilde{f}_{1}\right) \in \mathcal{H}^{(0,1)}$, имеет ненулевое решение, т.е. когда $\operatorname{det}\left[I^{(0,1)}+A_{0}(p ; z)\right]=0$. Лемма доказана.

Из леммы 3 вытекает, что число $z \in \mathbb{C} \backslash \sigma_{\mathrm{ess}}\left(h_{2}(p)\right)$ принадлежит дискретному спектру оператора $h_{2}(p)$ тогда и только тогда, когда $\operatorname{det}\left[I^{(0,1)}+A_{0}(p ; z)\right]=0$. Следовательно, верна следующая лемма.

ЛЕмма 4. Для дискретного спектра $\sigma_{\text {disc }}\left(h_{2}(p)\right)$ оператора $h_{2}(p), p \in \mathbf{T}^{\nu}$, uмеет место равенство

$$
\sigma_{\text {disc }}\left(h_{2}(p)\right)=\left\{z \in \mathbb{C} \backslash \sigma_{\text {ess }}\left(h_{2}(p)\right): \operatorname{det}\left[I^{(0,1)}+A_{0}(p ; z)\right]=0\right\} .
$$

Аналогично можно сформулировать и доказать следующую лемму.

Лемма 5. Для дискретного спектра $\sigma_{\text {disc }}\left(h_{1}(q)\right)$ оператора $h_{1}(q), q \in \mathbf{T}^{\nu}$, верно равенство

$$
\sigma_{\text {disc }}\left(h_{1}(q)\right)=\left\{z \in \mathbb{C} \backslash \sigma_{\mathrm{ess}}\left(h_{1}(q)\right): \operatorname{det}\left[I_{1}-k_{1}^{1 / 2} r_{0}(q ; z) k_{1}^{1 / 2}\right]=0\right\},
$$

где $r_{0}(q ; z)=\left(h_{1}^{(0)}(q)-z I_{1}\right)^{-1}-$ резольвента оператора $h_{1}^{(0)}(q), q \in \mathbf{T}^{\nu}$.

Теперь мы готовы доказать теорему 1.

ДокАЗАТЕЛЬСТво тЕОРЕмы 1. Применяя теорему о спектре разложимых операторов и учитывая равенства

$$
\begin{gathered}
\sigma\left(h_{i}(p)\right)=\sigma_{\mathrm{disc}}\left(h_{i}(p)\right) \cup[m(p), M(p)], \quad p \in \mathbf{T}^{\nu}, \quad i=1,2, \\
\bigcup_{p \in \mathbf{T}^{\nu}}[m(p), M(p)]=[m, M],
\end{gathered}
$$

мы приходим к утверждению теоремы 1.

\section{4. АНАЛОГ УРАВНЕНИЯ ФАДДЕЕВА ДЛЯ СОБСТВЕННЫХ ВЕКТОРОВ ОПЕРАТОРА $H$}

В этом разделе мы получим аналог уравнения Фаддеева для собственных векторов оператора $H$, который играет важную роль при изучении существенного и дискретного спектров рассматриваемого оператора. 
Пусть

$$
\overline{\mathcal{H}}_{0}=\mathcal{H}_{0}, \quad \overline{\mathcal{H}}_{1}=\mathcal{H}_{1}, \quad \overline{\mathcal{H}}_{2}=\overline{\mathcal{H}}_{3}=\mathcal{H}_{2}, \quad \overline{\mathcal{H}}=\bigoplus_{i=n}^{m} \overline{\mathcal{H}}_{i}
$$

Пусть операторные матрицы $A(z)$ и $K(z)$ при каждом $z \in \mathbb{C} \backslash \sigma_{\text {three }}\left(H_{2}\right)$ действуют в пространстве $\overline{\mathcal{H}}$ по формулам

$$
\begin{aligned}
A(z) & =\left(\begin{array}{cccc}
A_{00}(z) & 0 & 0 & 0 \\
0 & A_{11}(z) & 0 & A_{13}(z) \\
0 & 0 & A_{22}(z) & 0 \\
0 & A_{31}(z) & 0 & A_{33}(z)
\end{array}\right), \\
K(z) & =\left(\begin{array}{cccc}
K_{00}(z) & K_{01}(z) & 0 & 0 \\
K_{10}(z) & 0 & K_{12}(z) & 0 \\
0 & K_{21}(z) & 0 & K_{23}(z) \\
0 & 0 & K_{32}(z) & 0
\end{array}\right),
\end{aligned}
$$

где операторы $A_{i j}(z): \overline{\mathcal{H}}_{j} \rightarrow \overline{\mathcal{H}}_{i}, i, j=0,1,2,3$, определяются равенствами

$$
\begin{aligned}
\left(A_{00}(z) g_{0}\right)_{0} & =g_{0}, \quad\left(A_{11}(z) g_{1}\right)_{1}(p)=\left(w_{1}(p)-z-\int \frac{v_{2}^{2}(s) d s}{w_{2}(p, s)-z}\right) g_{1}(p), \\
\left(A_{13}(z) g_{3}\right)_{1}(p) & =\int \frac{v_{2}(s) d s}{w_{2}(p, s)-z} \int K_{2}^{1 / 2}(t, s) g_{3}(p, t) d t \\
\left(A_{22}(z) g_{2}\right)_{2}(p, q) & =g_{2}(p, q)-\left(K_{1}^{1 / 2} R_{22}^{0}(z) K_{1}^{1 / 2} g_{2}\right)(p, q), \\
\left(A_{31}(z) g_{1}\right)_{3}(p, q) & =\int \frac{K_{2}^{1 / 2}(s, q) v_{2}(s) d s}{w_{2}(p, s)-z} d s \cdot g_{1}(p), \\
\left(A_{33}(z) g_{3}\right)_{3}(p, q) & =g_{3}(p, q)-\left(K_{2}^{1 / 2} R_{22}^{0}(z) K_{2}^{1 / 2} g_{3}\right)_{3}(p, q),
\end{aligned}
$$

а операторы $K_{i j}(z): \overline{\mathcal{H}}_{j} \rightarrow \overline{\mathcal{H}}_{i}, i, j=0,1,2,3,-$ формулами

$$
\begin{aligned}
\left(K_{00}(z) g_{0}\right)_{0} & =\left(w_{0}-z+1\right) g_{0}, \quad K_{01}(z) \equiv H_{01}, \quad K_{10}(z) \equiv-H_{10} \\
\left(K_{12}(z) g_{2}\right)_{1}(p) & =\int \frac{v_{2}(s) d s}{w_{2}(p, s)-z} \int K_{1}^{1 / 2}(p, t) g_{2}(t, s) d t \\
\left(K_{21}(z) g_{1}\right)_{2}(p, q) & =-v_{2}(q) \int \frac{K_{1}^{1 / 2}(p, s) g_{1}(s) d s}{w_{2}(s, q)-z}, \\
\left(K_{23}(z) g_{3}\right)_{2}(p, q) & =\left(K_{1}^{1 / 2} R_{22}^{0}(z) K_{2}^{1 / 2} g_{3}\right)(p, q) \\
\left(K_{32}(z) g_{2}\right)_{3}(p, q) & =\left(K_{2}^{1 / 2} R_{22}^{0}(z) K_{1}^{1 / 2} g_{2}\right)(p, q) .
\end{aligned}
$$

Здесь $g_{i} \in \overline{\mathcal{H}}_{i}, i=0,1,2,3$; множество $\sigma_{\text {three }}\left(H_{2}\right)$ определено в теореме 1 и $R_{22}^{0}(z)=$ $\left(H_{22}^{0}-z I_{2}\right)^{-1}$ - резольвента оператора $H_{22}^{0}$.

Отметим, что операторы $K_{i j}(z), i, j=0,1,2,3$, при каждом $z \in \mathbb{C} \backslash \sigma_{\text {three }}\left(H_{2}\right)$ принадлежат пространству операторов Гильберта-Шмидта, следовательно, $K(z)$ является компактным оператором. 
Лемма 6. Oператор $A(z), z \in \mathbb{C} \backslash \sigma_{\text {three }}\left(H_{2}\right)$ является ограниченным и обратимьмм тогда и только тогда, когда $z \in \mathbb{C} \backslash\left(\sigma\left(H_{1}\right) \cup \sigma\left(H_{2}\right)\right)$.

ДокАзАтЕЛьство. Сначала определим матричный оператор $A_{0}(z)$, действующий в $\overline{\mathcal{H}}_{1} \oplus \overline{\mathcal{H}}_{3}$ как

$$
A_{0}(z)=\left(\begin{array}{ll}
A_{11}(z) & A_{13}(z) \\
A_{31}(z) & A_{33}(z)
\end{array}\right) .
$$

По определению операторов $A(z)$ и $A_{0}(z)$ имеем, что оператор $A(z)$ обратим тогда и только тогда, когда операторы $A_{0}(z)$ и $A_{22}(z)$ обратимы.

По аналогии с тем, как это делалось для оператора $H_{2}$, мы можем получить следующее разложение: $A_{0}(z)=\int \oplus\left[I^{(0,1)}+A_{0}(p ; z)\right] d p$, где оператор $A_{0}(p ; z)$ определен по формуле (3.3). В силу леммы 4 и теоремы 1 при любых фиксированных $p \in \mathbf{T}^{\nu}$ и $z \in \mathbb{C} \backslash \sigma\left(H_{2}\right)$ мы имеем, что $\operatorname{det}\left[I^{(0,1)}+A_{0}(p ; z)\right] \neq 0$. Следовательно, при каждом $z \in \mathbb{C} \backslash \sigma\left(H_{2}\right)$ оператор $A_{0}(z)$ является обратимым. Аналогично можно доказать, что при каждом $z \in \mathbb{C} \backslash \sigma\left(H_{1}\right)$ оператор $A_{22}(z)$ также является обратимым. Легко можно показать, что если операторы $A_{0}(z)$ и $A_{22}(z), z \in \mathbb{C} \backslash \sigma_{\text {three }}\left(H_{2}\right)$, являются ограниченными и обратимыми, то $z \in \mathbb{C} \backslash\left(\sigma\left(H_{1}\right) \cup \sigma\left(H_{2}\right)\right)$. Лемма доказана.

В силу леммы 6 при каждом $z \in \mathbb{C} \backslash\left(\sigma\left(H_{1}\right) \cup \sigma\left(H_{2}\right)\right)$ оператор $A(z)$ является ограниченным и обратимым, поэтому для таких $z$ мы можем определить оператор $T(z)=A^{-1}(z) K(z)$.

Следующая лемма устанавливает связь между собственными значениями операторов $H$ и $T(z)$.

Лемма 7. Число $z \in \mathbb{C} \backslash\left(\sigma\left(H_{1}\right) \cup \sigma\left(H_{2}\right)\right)$ является собственным значением оператора $H$ тогда и толъко тогда, когда число $\lambda=1$ является собственным значением onepamopa $T(z)$.

ДоказАтельство. Пусть $z \in \mathbb{C} \backslash\left(\sigma\left(H_{1}\right) \cup \sigma\left(H_{2}\right)\right)$ - собственное значение оператора $H$. Тогда уравнение $H f=z f$ или, что эквивалентно, система уравнений

$$
\begin{aligned}
\left(\left(H_{00}-z I_{0}\right) f_{0}\right)_{0}+\left(H_{01} f_{1}\right)_{0} & =0 \\
\left(H_{10} f_{0}\right)_{1}(p)+\left(\left(H_{11}-z I_{1}\right) f_{1}\right)_{1}(p)+\left(H_{12} f_{2}\right)_{1}(p) & =0 \\
\left(H_{21} f_{1}\right)_{2}(p, q)+\left(\left(H_{22}-z I_{2}\right) f_{2}\right)_{2}(p, q) & =0
\end{aligned}
$$

имеет нетривиальное решение $f=\left(f_{0}, f_{1}, f_{2}\right) \in \mathcal{H}^{(0,2)}$. Так как $z \notin \sigma_{\text {three }}\left(H_{1}\right)$, то из третьего уравнения системы (4.1) для $f_{2}$ имеем

$$
f_{2}(p, q)=\left(R_{22}^{0}(z) K_{1} f_{2}\right)(p, q)+\left(R_{22}^{0}(z) K_{2} f_{2}\right)(p, q)-\left(R_{22}^{0}(z) H_{21} f_{1}\right)(p, q),
$$

где $R_{22}^{0}(z)=\left(H_{22}^{0}-z I_{2}\right)^{-1}-$ резольвента оператора $H_{22}^{0}$.

Пусть

$$
\psi_{i}(p, q)=\left(K_{i}^{1 / 2} f_{2}\right)(p, q), \quad i=1,2 .
$$

Тогда равенство (4.2) можно переписать в виде

$$
f_{2}(p, q)=\left(R_{22}^{0}(z) K_{1}^{1 / 2} \psi_{1}\right)(p, q)+\left(R_{22}^{0}(z) K_{2}^{1 / 2} \psi_{2}\right)(p, q)-\left(R_{22}^{0}(z) H_{21} f_{1}\right)(p, q) .
$$


Подставляя это выражение во второе уравнение системы (4.1) и равенство (4.3), получим, что система уравнений

$$
\begin{gathered}
f_{0}=\left(w_{0}-z+1\right) f_{0}+\int v_{1}(s) f_{1}(s) d s \\
\left(w_{1}(p)-z-\int \frac{v_{2}^{2}(s) d s}{w_{2}(p, s)-z}\right) f_{1}(p)+\int \frac{v_{2}(s) d s}{w_{2}(p, s)-z} \int K_{2}^{1 / 2}(t, s) \psi_{2}(p, t) d t= \\
=-v_{1}(p) f_{0}-\int \frac{v_{2}(s) d s}{w_{2}(p, s)-z} \int K_{1}^{1 / 2}(p, t) \psi_{1}(t, s) d t \\
\psi_{1}(p, q)-\left(K_{1}^{1 / 2} R_{22}^{0}(z) K_{1}^{1 / 2} \psi_{1}\right)(p, q)= \\
=-v_{2}(q) \int \frac{K_{1}^{1 / 2}(p, s) f_{1}(s) d s}{w_{2}(s, q)-z}+\left(K_{1}^{1 / 2} R_{22}^{0}(z) K_{2}^{1 / 2} \psi_{2}\right)(p, q), \\
\left(K_{2}^{1 / 2} R_{22}^{0}(z) H_{21} f_{1}\right)(p, q)+\psi_{2}(p, q)-\left(K_{2}^{1 / 2} R_{22}^{0}(z) K_{2}^{1 / 2} \psi_{2}\right)(p, q)= \\
=\left(K_{2}^{1 / 2} R_{22}^{0}(z) K_{1}^{1 / 2} \psi_{1}\right)(p, q)
\end{gathered}
$$

или уравнение $A(z) \psi=K(z) \psi, \psi=\left(f_{0}, f_{1}, \psi_{1}, \psi_{2}\right) \in \overline{\mathcal{H}}$, имеют ненулевое решение тогда и только тогда, когда система уравнений (4.1) имеет ненулевое решение.

В силу леммы 6 при каждом фиксированном $z \notin \sigma_{\text {two }}\left(H_{1}\right) \cup \sigma_{\text {two }}\left(H_{2}\right)$ оператор $A(z)$ является обратимым и, следовательно, уравнение $\psi=A^{-1}(z) K(z) \psi$, или $\psi=T(z) \psi$, имеет нетривиальное решение тогда и только тогда, когда система уравнений (4.1) имеет ненулевое решение. Лемма доказана.

ЗАмЕчАниЕ 2. Уравнение $T(z) \psi=\psi$ является аналогом системы интегральных уравнений типа уравнений Фаддеева для собственных векторов оператора $H$.

\section{5. ДОКАЗАТЕЛЬСТВО ОСНОВНОГО РЕЗУЛЬТАТА}

В этом разделе, пользуясь утверждениями разделов 3 и 4, а также критерием Вейля, мы докажем основной результат работы.

ДоказАТЕЛЬСтво теоремы 2. Сначала докажем, что $\sigma\left(H_{1}\right) \cup \sigma\left(H_{2}\right) \subset \sigma_{\text {ess }}(H)$. С этой целью перепишем множество $\sigma\left(H_{1}\right) \cup \sigma\left(H_{2}\right)$ в виде

$$
\sigma\left(H_{1}\right) \cup \sigma\left(H_{2}\right)=\sigma_{\text {two }}\left(H_{1}\right) \cup \sigma_{\text {two }}\left(H_{2}\right) \cup \sigma_{\text {three }}\left(H_{2}\right) .
$$

Включение $\sigma_{\text {three }}\left(H_{2}\right) \subset \sigma_{\text {ess }}(H)$ доказывается аналогично тому, как это сделано в работе [11].

Докажем, что $\sigma_{\text {two }}\left(H_{2}\right) \subset \sigma_{\text {ess }}(H)$. Для этого удобно воспользоваться критерием Вейля, т.е. достаточно построить последовательность ортонормированных векторов $\left\{f^{(n)}\right\}$, для которых $\left\|\left(H-z_{0}\right) f^{(n)}\right\| \rightarrow 0$ при $n \rightarrow \infty$. Пусть $z_{0} \in \sigma_{\text {two }}\left(H_{2}\right)-$ произвольная точка. Тогда по лемме 4 существует $p_{0} \in \mathbf{T}^{\nu}$ такое, что $z_{0} \in \sigma_{\text {disc }}\left(h_{2}\left(p_{0}\right)\right)$. Следовательно, существует ненулевой вектор $\varphi=\left(\varphi_{0}, \varphi_{1}\right) \in \mathcal{H}^{(0,1)}$ такой, что

$$
\left(h_{2}\left(p_{0}\right)-z_{0} I^{(0,1)}\right) \varphi=0 .
$$


Положим $f^{(n)}=\left(0, f_{1}^{(n)}, f_{2}^{(n)}\right) \in \mathcal{H}^{(0,2)}$, где

$$
f_{1}^{(n)}(p)=\frac{\chi_{V_{n}}(p)}{\sqrt{\mu\left(V_{n}\left(p_{0}\right)\right)}} \frac{\varphi_{0}}{\|\varphi\|}, \quad f_{2}^{(n)}(p, q)=\frac{\chi_{V_{n}}(p)}{\sqrt{\mu\left(V_{n}\left(p_{0}\right)\right)}} \frac{\varphi_{1}(q)}{\|\varphi\|},
$$

$\chi_{V_{n}}(\cdot)$ есть характеристическая функция множества

$$
V_{n}\left(p_{0}\right)=\left\{p \in \mathbf{T}^{\nu}: \frac{1}{n+1}<\left|p-p_{0}\right|<\frac{1}{n}\right\},
$$

$\mu\left(V_{n}\left(p_{0}\right)\right)$ - лебегова мера множества $V_{n}\left(p_{0}\right)$. Легко можно проверить, что система $\left\{f^{(n)}\right\}$ является ортонормированной. Покажем, что при $z_{0} \in \sigma_{\text {two }}\left(H_{2}\right)$

$$
\lim _{n \rightarrow \infty}\left\|\left(H-z_{0} I^{(0,2)}\right) f^{(n)}\right\|=0, \quad I^{(0,2)}=\operatorname{diag}\left\{I_{0}, I_{1}, I_{2}\right\} .
$$

Заметим, что

$$
\begin{aligned}
\left\|\left(H-z_{0} I^{(0,2)}\right) f^{(n)}\right\|^{2}= & \frac{\left|\varphi_{0}\right|^{2}}{\|\varphi\|^{2}} \cdot\left|\int \frac{\chi_{V_{n}}(s) v_{1}(s) d s}{\sqrt{\mu\left(V_{n}\left(p_{0}\right)\right)}}\right|^{2}+ \\
& +\int\left|\int \frac{\chi_{V_{n}}(s) K_{1}(p, s) d s}{\sqrt{\mu\left(V_{n}\left(p_{0}\right)\right)}}\right|^{2} d p \int \frac{\left|\varphi_{1}(q)\right|^{2} d q}{\|\varphi\|^{2}}+ \\
& +\int\left|\left(\left(H_{11}-z_{0} I_{1}\right) f_{1}^{(n)}\right)_{1}(p)+\left(H_{12} f_{2}^{(n)}\right)_{1}(p)\right|^{2} d p+ \\
& +\int\left|\left(H_{21} f_{1}^{(n)}\right)_{2}(p, q)+\left(\left(H_{22}-z_{0} I_{2}\right) f_{2}^{(n)}\right)_{2}(p, q)\right|^{2} d p d q .
\end{aligned}
$$

Оценим сумму двух первых слагаемых в правой части равенства величиной $C \sqrt{\mu\left(V_{n}\left(p_{0}\right)\right)}$, которая по построению множества $V_{n}\left(p_{0}\right)$ стремится к нулю при $n \rightarrow \infty$. Сумму двух оставшихся слагаемых оценим величиной

$$
\int \frac{\chi_{V_{n}}(p)}{\mu\left(V_{n}\left(p_{0}\right)\right)}\left\|h_{2}(p)-z_{0} I^{(0,1)}\right\|^{2} d p \leqslant \max _{p \in V_{n}\left(p_{0}\right)}\left\|h_{2}(p)-z_{0} I^{(0,1)}\right\|^{2} .
$$

В силу равенства (5.1) последнее выражение стремится к нулю при $n \rightarrow \infty$. Из произвольности точки $z_{0} \in \sigma_{\text {two }}\left(H_{2}\right)$ вытекает, что $\sigma_{\text {two }}\left(H_{2}\right) \subset \sigma_{\text {ess }}(H)$.

Включение $\sigma_{\text {two }}\left(H_{1}\right) \subset \sigma_{\text {ess }}(H)$ доказывается аналогично. Таким образом, мы доказали, что $\left(\sigma\left(H_{1}\right) \cup \sigma\left(H_{2}\right)\right) \subset \sigma_{\text {ess }}(H)$.

Теперь докажем обратное включение, т.е. покажем, что $\sigma_{\mathrm{ess}}(H) \subset\left(\sigma\left(H_{1}\right) \cup \sigma\left(H_{2}\right)\right)$. В силу компактности оператора $K(z)$ и ограниченности оператора $A^{-1}(z)$ получаем, что $T(z)$ - компактная операторнозначная аналитическая функция на множестве $\mathbb{C} \backslash\left(\sigma\left(H_{1}\right) \cup \sigma\left(H_{2}\right)\right)$ и оператор $I-T(z)$ обратим при больших $|z|$, где $z$ вещественное число (здесь $I$ - единичный оператор в $\overline{\mathcal{H}}$ ). Согласно аналитической теореме Фредгольма [2] существует дискретное множество $S \subset \mathbb{C} \backslash\left(\sigma\left(H_{1}\right) \cup\right.$ $\left.\sigma\left(H_{2}\right)\right)$ такое, что операторнозначная функция $(I-T(z))^{-1}$ существует и аналитична в $\mathbb{C} \backslash\left(\sigma\left(H_{1}\right) \cup \sigma\left(H_{2}\right) \cup S\right)$, а в точках $S$ имеет вычеты конечного ранга. Следовательно, в силу леммы 7 для оператора $H-z I^{(0,2)}$ существует ограниченный обратный 
оператор. Это означает, что множество $\sigma(H) \backslash\left(\sigma\left(H_{1}\right) \cup \sigma\left(H_{2}\right)\right)$ состоит только из изолированных точек и может иметь предельные точки только на границе множества $\sigma\left(H_{1}\right) \cup \sigma\left(H_{2}\right)$. Следовательно, $\sigma(H) \backslash\left(\sigma\left(H_{1}\right) \cup \sigma\left(H_{2}\right)\right) \subset \sigma(H) \backslash \sigma_{\mathrm{ess}}(H)$, т.е. $\sigma_{\text {ess }}(H) \subset\left(\sigma\left(H_{1}\right) \cup \sigma\left(H_{2}\right)\right)$. Теорема 2 доказана.

Благодарности. Автор выражает искреннюю благодарность М.Э. Муминову за плодотворные обсуждения результатов работы и рецензенту за ценные и полезные замечания.

\section{Список литературы}

[1] Г. М. Жислин, Труды ММО, 9, 1960, 81-120.

[2] М. Рид, Б. Саймон, Методы современной математической физики. Т. 4. Анализ операторов, Мир, М., 1982.

[3] С. Н. Лакаев, М. Э. Муминов, ТМФ, 135:3 (2003), 478-503.

[4] М.Э. Муминов, ТМФ, 148:3 (2006), 428-443.

[5] R. A. Minlos, H. Spohn, "The three-body problem in radioactive decay: the case of one atom and at most two photons", Topics in Statistical and Theoretical Physics, Amer. Math. Soc. Transl. Ser. 2, 177, ed. R. L. Dobrushin, AMS, Providence, RI, 1996, 159-193.

[6] Ю. В. Жуков, Р. А. Минлос, ТМФ, 103:1 (1995), 63-81.

[7] Г. Р. Ёдгоров, М. Э. Муминов, ТМФ, 144:3 (2005), 544-554.

[8] Т. Х. Расулов, Матем. заметки, 83:1 (2008), 86-94.

[9] M. I. Muminov, T. H. Rasulov, The Faddeev Equation and Essential Spectrum of a Hamiltonian in Fock Space, ICTP Preprint IC/2008/027.

[10] Т. Х. Расулов, Изв. вузов. Матем., 2008, № 12, 59-69.

[11] С. Н. Лакаев, Т. Х. Расулов, Матем. заметки, 73:4 (2003), 556-564.

[12] С. Н. Лакаев, Т. Х. Расулов, Функи. анализ и его прил., 37:1 (2003), 81-84.

[13] S. Albeverio, S. N. Lakaev, T. H. Rasulov, J. Stat. Phys., 127:2 (2007), 191-220.

[14] Т. Х. Расулов, ТМФ, 152:3 (2007), 518-527.

Поступила в редакцию 17.11.2008, после доработки 12.02.2009 